Proyecciones Journal of Mathematics

Vol. 38, No 2, pp. 191-202, June 2019.

Universidad Católica del Norte

Antofagasta - Chile

\title{
Further results on 3-product cordial labeling
}

\author{
P. Jeyanthi \\ Govindammal Aditanar College for Women, India \\ A. Maheswari \\ Kamaraj College of Engineering and Technology, India \\ and \\ M. Vijayalakshmi \\ Dr. G. U. Pope College of Engineering, India \\ Received : November 2015. Accepted : November 2018
}

\begin{abstract}
A mapping $f: V(G) \rightarrow\{0,1,2\}$ is called 3-product cordial labeling if $\left|v_{f}(i)-v_{f}(j)\right| \leq 1$ and $\left|e_{f}(i)-e_{f}(j)\right| \leq 1$ for any $i, j \in\{0,1,2\}$, where $v_{f}(i)$ denotes the number of vertices labeled with $i, e_{f}(i)$ denotes the number of edges xy with $f(x) f(y) \equiv i(\bmod 3)$. A graph with 3product cordial labeing is called 3-product cordial graph. In this paper we establish that switching of an apex vertex in closed helm, double fan, book graph $K_{1, n} \times K_{2}$ and permutation graph $P\left(K_{2}+m K_{1}, I\right)$ are 3-product cordial graphs.
\end{abstract}

Key Words. cordial labeling, product cordial labeling, 3-product cordial labeling, 3-product cordial graph.

AMS Subject Classification (2010) : 05C78 


\section{Introduction}

All graphs considered here are simple, finite, connected and undirected. For basic notations and terminology, we follow [3]. A graph labeling is an assignment of integers to the vertices or edges or both, subject to certain conditions. There are several types of labeling and a complete survey of graph labeling is available in [2]. Cordial labeling is a weaker version of graceful labeling and harmonious labeling introduced by Cahit in [1]. Let $f$ be a function from the vertices of $G$ to $\{0,1\}$ and for each edge $x y$ assign the label $|f(x)-f(y)| . f$ is called a cordial labeling of $G$ if the number of vertices labeled 0 and the number of vertices labeled 1 differ by at most 1 , and the number of edges labeled 0 and the number of edges labeled 1 differ at most by 1 . Sundaram et al. introduced the concept of product cordial labeling in [10]. Let $f$ be a function from $V(G)$ to $\{0,1\}$. For each edge $u v$, assign the label $f(u) f(v)$. Then $f$ is called product cordial labeling if $\left|v_{f}(0)-v_{f}(1)\right| \leq 1$ and $\left|e_{f}(0)-e_{f}(1)\right| \leq 1$ where $v_{f}(i)$ and $e_{f}(i)$ denotes the number of vertices and edges respectively labeled with $i(i=0,1)$. The same authors have introduced the concept of EP-cordial labeling in [11]. A vertex labeling $f: V(G) \rightarrow\{-1,0,1\}$ is said to be an EP-cordial labeling if it induces the edge labeling $f^{*}$ defined by $f^{*}(u v)=f(u) f(v)$ for each $u v \in E(G)$ and if $\left|v_{f}(i)-v_{f}(j)\right| \leq 1$ and $\left|e_{f}(i)-e_{f}(j)\right| \leq 1$ for any $i \neq j i, j \in\{-1,0,1\}$, where $v_{f}(x)$ and $e_{f}(x)$ denotes the number of vertices and edges of $G$ having the label $x \in\{-1,0,1\}$. In [11] it is remarked that any EP-cordial labeling is a 3-product cordial labeling. A mapping $f: V(G) \rightarrow\{0,1,2\}$ is called 3-product cordial labeling if $\left|v_{f}(i)-v_{f}(j)\right| \leq 1$ and $\left|e_{f}(i)-e_{f}(j)\right| \leq 1$ for any $i, j \in\{0,1,2\}$, where $v_{f}(i)$ denotes the number of edges $x y$ with $f(x) f(y)=i(\bmod 3)$. A graph with 3-product cordial labeling is called a 3-product cordial graph. Jeyanthi and Maheswari [4]-[8] proved that the graphs $\left\langle B_{n, n}: w\right\rangle, C_{n} \cup P_{n}, C_{m} \circ \overline{K_{n}}$ if $m \geq 3$ and $n \geq 1, P_{m} \circ \overline{K_{n}}$ if $m, n \geq 1$, duplicating arbitrary vertex in cycle $C_{n}$, duplicating arbitrary edge in cycle $C_{n}$, duplicating arbitrary vertex in wheel $W_{n}$, middle graph of $P_{n}$, the splitting graph of $P_{n}$, the total graph of $P_{n}, P_{n}\left[P_{2}\right], p_{n}^{2}, K_{2, n}$, vertex switching of $C_{n}$, ladder $L_{n}$, triangular ladder $T L_{n}$, the graph $\left\langle w_{n}^{(1)}: w_{n}^{(2)}: \ldots: w_{n}^{(k)}\right\rangle$, the splitting graphs $S^{\prime}\left(K_{1, n}\right), S^{\prime}\left(B_{n, n}\right)$, the shadow graph $D_{2}\left(B_{n, n}\right)$, the square graph $B_{n, n}^{2}$, triangular snake, double alternate triangular snake and alternate triangular snake graphs are 3-product cordial graphs. Also they proved that a complete graph $K_{n}$ is a 3-product cordial graph if and only if $n \leq 2$.

In addition, they proved that if $G(p, q)$ is a 3-product cordial graph (i) 
$p \equiv 1(\bmod 3)$ then $q \leq \frac{p^{2}-2 p+7}{3}$. (ii) $p \equiv 2(\bmod 3)$ then $q \leq \frac{p^{2}-p+4}{3}$ (iii) $p \equiv 0(\bmod 3)$ then $q \leq \frac{p^{2}-3 p+6}{3}$ and if $G_{1}$ is a 3 -product cordial graph with $3 m$ vertices and $3 n$ edges and $G_{2}$ is any 3-product cordial graph then $G_{1} \cup G_{2}$ is also 3-product cordial graph. In this paper we establish that switching of an apex vertex in closed helm, double fan, $K_{1, n} \times K_{2}$ and permutation graph $P\left(K_{2}+m K_{1}, I\right)$ are 3-product cordial graphs. We use the following definitions in the subsequent section.

Definition 1.1. The vertex switching $G_{v}$ of a graph $G$ is the graph obtained by taking a vertex $v$ of $G$, by removing all the edges incident with $v$ and joining the vertex $v$ to every vertex which is not adjacent to $v$ in $G$.

Definition 1.2. The helm $H_{n}$ is the graph obtained from a wheel $W_{n}$ by attaching a pendant edge to each rim vertex.

Definition 1.3. The closed helm $\mathrm{CH}_{n}$ is the graph obtained from a helm $H_{n}$ by joining each pendant vertex to from a cycle.

Definition 1.4. The graph $P_{n}+2 K_{1}$ is called a double fan $D F_{n}$.

Definition 1.5. For any permutation $f$ on $1,2,3, \ldots, n$, the $f$-permutation graph on a graph $G, P(G, f)$ consists of two disjoint copies of $G$, say $G_{1}$ and $G_{2}$, each of which has vertices labeled $v_{1}, v_{2}, \ldots, v_{n}$ with $n$ edges obtained by joining each $v_{i}$ in $G_{1}$ to $v_{f(i)}$ in $G_{2}$. We denote the identity permutation by $I$.

For any real number $n,\lceil n\rceil$ denotes the smallest integer $\geq n$ and $\lfloor n\rfloor$ denotes the greatest integer $\leq n$.

\section{Main Results}

Theorem 2.1. The graph obtained by switching of an apex vertex in closed helm $\mathrm{CH}_{n}$ admits 3-product cordial labeling if and only if $n \equiv$ $2(\bmod 3)$.

Proof. Let $v$ be the apex vertex $v_{1}, v_{2}, v_{3}, \ldots, v_{n}$ be the vertices of inner cycle and $u_{1}, u_{2}, u_{3}, \ldots, u_{n}$ be the vertices of outer cycle $C H_{n}$. Let $G_{v}$ denotes graph obtained by switching of an apex vertex $v$ of $G=C H_{n}$. Then $\left|V\left(G_{v}\right)\right|=2 n+1$ and $\left|E\left(G_{v}\right)\right|=4 n$. We define $f: V\left(G_{v}\right) \rightarrow\{0,1,2\}$ as follows: 
$f(v)=2$, For $1 \leq i \leq\left\lfloor\frac{2 n+1}{3}\right\rfloor, f\left(v_{i}\right)=0$.

For $n \equiv 0,2,3(\bmod 4), 1 \leq i \leq\left\lceil\frac{n}{3}\right\rceil$,

$f\left(v_{\left\lfloor\frac{2 n+1}{3}\right\rfloor+i}\right)= \begin{cases}1 & \text { for } i \equiv 1,2(\bmod 4) \\ 2 & \text { for } i \equiv 0,3(\bmod 4)\end{cases}$

For $n \equiv 1(\bmod 4), n>5,1 \leq i \leq\left\lceil\frac{n}{3}\right\rceil-2$,

$f\left(v_{\left\lfloor\frac{2 n+1}{3}\right\rfloor+i}\right)= \begin{cases}1 & \text { for } i \equiv 1,2(\bmod 4) \\ 2 & \text { for } i \equiv 0,3(\bmod 4)\end{cases}$

$f\left(v_{n-1}\right)=1$ and $f\left(v_{n}\right)=2$.

For $n=5, f\left(v_{4}\right)=1$ and $f\left(v_{5}\right)=2$.

For $n \equiv 0,1,3(\bmod 4), 1 \leq i \leq n, f\left(u_{i}\right)= \begin{cases}1 & \text { if } i \equiv 1,2(\bmod 4) \\ 2 & \text { if } i \equiv 0,3(\bmod 4)\end{cases}$

For $n \equiv 2(\bmod 4), 1 \leq i \leq n-2, f\left(u_{i}\right)= \begin{cases}1 & \text { if } i \equiv 1,2(\bmod 4) \\ 2 & \text { if } i \equiv 0,3(\bmod 4)\end{cases}$

$f\left(u_{n-1}\right)=1$ and $f\left(u_{n}\right)=2$.

In view of the above labeling pattern we have $v_{f}(0)+1=v_{f}(1)=$ $v_{f}(2)=\left\lceil\frac{2 n+1}{3}\right\rceil, e_{f}(0)=e_{f}(1)=e_{f}(2)+1=\left\lceil\frac{4 n}{3}\right\rceil$ if $n \equiv 0,1(\bmod 4)$ and $e_{f}(0)=e_{f}(1)+1=e_{f}(2)=\left\lceil\frac{4 n}{3}\right\rceil$ if $n \equiv 2,3(\bmod 4)$.

Thus we have $\left|v_{f}(i)-v_{f}(j)\right| \leq 1$ and $\left|e_{f}(i)-e_{f}(j)\right| \leq 1$ for all $i, j=$ $0,1,2$. Hence $f$ is a 3 -product cordial labeling of $G_{v}$ if $n \equiv 2(\bmod 3)$.

Conversely, we assume that $n \equiv 0(\bmod 3)$ and take $n=3 k$. Then $\left|V\left(G_{v}\right)\right|=6 k+1$ and $\left|E\left(G_{v}\right)\right|=12 k$.

Let $f$ be a 3 -product cordial labeling of $G_{v}$. Hence we have $v_{f}(0)=$ $v_{f}(1)-1=v_{f}(2)=2 k$ or $v_{f}(0)=v_{f}(1)=v_{f}(2)-1=2 k$ and $e_{f}(0)=$ $e_{f}(1)=e_{f}(2)=4 k$. If $f\left(u_{i}\right)=0$ if $1 \leq i \leq 2 k$, then $e_{f}(0)=6 k+1$. If $f\left(v_{i}\right)=0$ if $1 \leq i \leq 2 k$, then $e_{f}(0)=4 k+1$. If $f\left(u_{i}\right)=0$ if $1 \leq i \leq 2 k-1$ and $f(v)=0$, then $e_{f}(0)=7 k-1$. If $f\left(v_{i}\right)=0$ if $1 \leq i \leq 2 k-1$ and $f(v)=0$, then $e_{f}(0)=7 k-1$. Thus, none of $f\left(u_{i}\right), f\left(v_{i}\right)$ and $f(v)$ is 0 . From the above argument, we get a contradiction to, $f$ is a 3 -product cordial labeling. Hence $G_{v}$ is not a 3 -product cordial graph if $n \equiv 0(\bmod 3)$.

We assume that $n \equiv 1(\bmod 3)$ and take $n=3 k+1$. Then $\left|V\left(G_{v}\right)\right|=$ $6 k+3$ and $\left|E\left(G_{v}\right)\right|=12 k+4$.

Let $f$ be a 3 -product cordial labeling of $G_{v}$. Hence we have $v_{f}(0)=$ $v_{f}(1)=v_{f}(2)=2 k+1$ and $e_{f}(0)=4 k+1$ or $4 k+2$. If $v_{f}(0)=2 k+1$, we assign 0 to $2 k+1$ vertices of degree 3 . We get $e_{f}(0)=4 k+3$. we assign 
0 to $2 k+1$ vertices of degree 4 . We get $e_{f}(0)=6 k+4$. If $f\left(u_{i}\right)=0$ if $1 \leq i \leq 2 k$ and $f(v)=0$, then $e_{f}(0)=7 k+2$. If $f\left(v_{i}\right)=0$ if $1 \leq i \leq 2 k$ and $f(v)=0$, then $e_{f}(0)=7 k+2$. Thus, none of $f\left(u_{i}\right), f\left(v_{i}\right)$ and $f(v)$ is 0 . From the above argument, we get a contradiction to, $f$ is a 3-product cordial labeling. Hence $G_{v}$ is not a 3 -product cordial graph if $n \equiv 1(\bmod 3)$.

An example for the 3-product cordial labeling of a closed helm $\mathrm{CH}_{8}$ by switching of an apex vertex is shown in Figure 1.

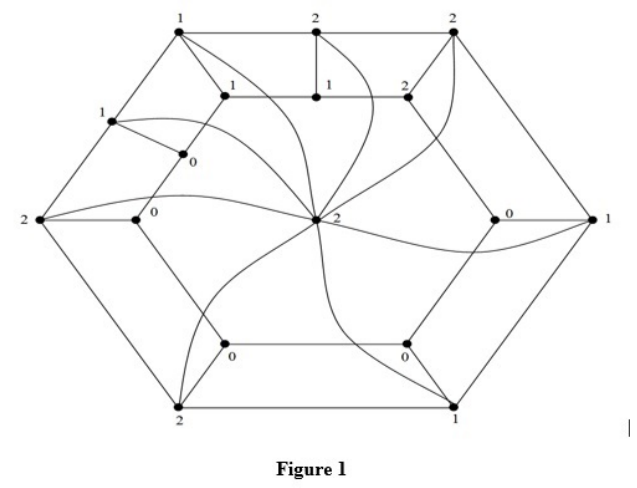

Theorem 2.2. The double fan graph $D F_{n}$ is a 3-product cordial graph if and only if $n \equiv 0(\bmod 3)$.

Proof. Let $D F_{n}$ be the double fan with apex vertices $u, v$ and $v_{1}, v_{2}, \ldots, v_{n}$ be the vertices of common path. Then $\left|V\left(D F_{n}\right)\right|=n+2$ and $\left|E\left(D F_{n}\right)\right|=$ $3 n-1$. To define $f: V\left(D F_{n}\right) \rightarrow\{0,1,2\}$ as follows:

Let $f(u)=1, f(v)=2, f\left(v_{i}\right)=0$ if $1 \leq i \leq \frac{n}{3}$.

For $n$ is even, $i=\frac{n}{3}+j, 1 \leq j \leq \frac{2 n}{3}, f\left(v_{i}\right)= \begin{cases}1 & \text { if } j \equiv 1,2(\bmod 4) \\ 2 & \text { if } j=0,3(\bmod 4)\end{cases}$

For $n$ is odd, $i=\frac{n}{3}+j, 1 \leq j \leq \frac{2 n}{3}-2, f\left(v_{i}\right)= \begin{cases}1 & \text { if } j \equiv 1,2(\bmod 4) \\ 2 & \text { if } j=0,3(\bmod 4)\end{cases}$

$f\left(v_{n-1}\right)=1, f\left(v_{n}\right)=2$.

In view of the above labeling pattern we have $v_{f}(0)+1=v_{f}(1)=$ $v_{f}(2)=\frac{n}{3}+1$ and $e_{f}(0)=e_{f}(1)=e_{f}(2)+1=n$ if $n$ is even, $e_{f}(0)=$ $e_{f}(1)+1=e_{f}(2)=n$ if $n$ if $n$ is odd.

Hence, we have $\left|v_{f}(i)-v_{f}(j)\right| \leq 1$ and $\left|e_{f}(i)-e_{f}(j)\right| \leq 1$ for all $i, j=$ $0,1,2$. Thus, $f$ is a 3 -product cordial labeling. Therefore, $D F_{n}$ is a 3 product cordial graph if $n \equiv 0(\bmod 3)$.

Conversely, we assume that $n \equiv 2(\bmod 3)$ and take $n=3 k+2$. Then $\left|V\left(D F_{n}\right)\right|=3 k+4$ and $\left|E\left(D F_{n}\right)\right|=9 k+5$. 
Let $f$ be a 3 -product cordial labeling of $D F_{n}$. Hence we have $v_{f}(0)=$ $v_{f}(1)-1=v_{f}(2)=k+1$ or $v_{f}(0)=v_{f}(1)=v_{f}(2)-1=k+1$ and $e_{f}(0)=3 k+1$ or $3 k+2$. If $f(u)=0, f(v)=0$ and the remaining $k-1$ vertices are $f\left(v_{i}\right)=0$ then we get, $e_{f}(0)>7 k+3$. If $f(u)=0$ or $f(v)=0$ and the remaining $k$ vertices are $f\left(v_{i}\right)=0$ for $1 \leq i \leq k$ then we get, $e_{f}(0)=5 k+2$. If $f\left(v_{i}\right)=0$ for $1 \leq i \leq k+1$ then we get $e_{f}(0)=3 k+3$. From the above argument, we get a contradiction to, $f$ is a 3 -product cordial labeling. Hence $D F_{n}$ is not a 3 -product cordial graph if $n \equiv 2(\bmod 3)$.

We assume that $n \equiv 1(\bmod 3)$ and take $n=3 k+1$. Then $\left|V\left(D F_{n}\right)\right|=$ $3 k+3$ and $\left|E\left(D F_{n}\right)\right|=9 k+2$.

Let $f$ be a 3-product cordial labeling of $D F_{n}$. Hence we have $v_{f}(0)=$ $v_{f}(1)=v_{f}(2)=k+1$ and $e_{f}(0)=3 k$ or $3 k+1$. If $f(u)=0, f(v)=0$ and the remaining $k-1$ vertices are $f\left(v_{i}\right)=0$ then we get, $e_{f}(0)>7 k+1$. If $f(u)=0$ or $f(v)=0$ and the remaining $k$ vertices are $f\left(v_{i}\right)=0$ for $1 \leq i \leq k$ then we get, $e_{f}(0)=5 k+1$. If $f\left(v_{i}\right)=0$ for $1 \leq i \leq k+1$ then we get $e_{f}(0)=3 k+3$. From the above argument, we get a contradiction to, $f$ is a 3-product cordial labeling. Hence $D F_{n}$ is not a 3-product cordial graph if $n \equiv 1(\bmod 3)$.

An example for the 3 -product cordial labeling for the graph $D F_{6}$ is shown in Figure 2.

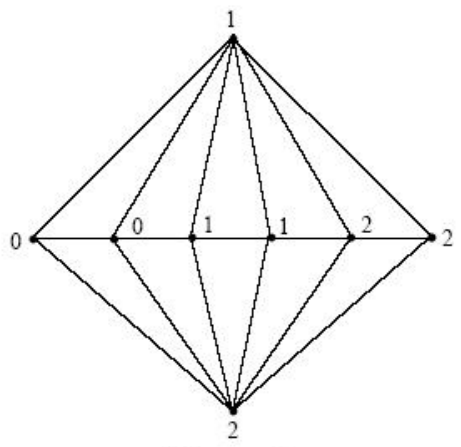

Figure 2.

Theorem 2.3. The book graph $K_{1, n} \times K_{2}$ is a 3 -product cordial graph.

Proof. Let the vertices of $K_{1, n} \times K_{2}$ be $\left\{u, v, u_{1}, u_{2}, \ldots, u_{n}, v_{1}, v_{2}, \ldots, v_{n}\right\}$ and the edges are $\{u v\} \cup\left\{u_{i} v_{i} / 1 \leq i \leq n\right\} \cup\left\{u u_{i} / 1 \leq i \leq n\right\}$. Clearly $K_{1, n} \times K_{2}$ has $2 n+2$ vertices and $3 n+1$ edges. Define $f: V\left(K_{1, n} \times K_{2}\right) \rightarrow$ $\{0,1,2\}$ by the following cases.

$$
f(u)=1, f(v)=2 \text {. }
$$


Case (i): $n \equiv 0(\bmod 3), n=3 k, k>1$.

For $\mathrm{k}$ is even, $\mathrm{f}\left(\mathrm{u}_{i}\right)= \begin{cases}0 & \text { if } 1 \leq i \leq k \\ 2 & \text { if } k+1 \leq i \leq \frac{3 k}{2} \\ 1 & \text { if } \frac{3 k}{2}+1 \leq i \leq 3 k\end{cases}$

and $f\left(\mathrm{v}_{i}\right)= \begin{cases}0 & \text { if } 1 \leq i \leq k \\ 1 & \text { if } k+1 \leq i \leq \frac{3 k}{2} \\ 2 & \text { if } \frac{3 k}{2}+1 \leq i \leq 3 k\end{cases}$

For $\mathrm{k}$ is odd, $\mathrm{f}\left(\mathrm{u}_{i}\right)= \begin{cases}0 & \text { if } 1 \leq i \leq k \\ 2 & \text { if } k+1 \leq i<\left\lceil\frac{3 k}{2}\right\rceil \\ 1 & \text { if }\left\lceil\frac{3 k}{2}\right\rceil \leq i \leq 3 k\end{cases}$

and $f\left(v_{i}\right)= \begin{cases}0 & \text { if } 1 \leq i \leq k \\ 1 & \text { if } k+1 \leq i<\left\lceil\frac{3 k}{2}\right\rceil \\ 2 & \text { if }\left\lceil\frac{3 k}{2}\right\rceil \leq i \leq 3 k\end{cases}$

For $k=1, f\left(u_{1}\right)=f\left(v_{1}\right)=0, f\left(u_{2}\right)=f\left(u_{3}\right)=1, f\left(v_{2}\right)=f\left(v_{3}\right)=2$.

From the above labeling we have, $v_{f}(0)+1=v_{f}(1)=v_{f}(2)=\left\lceil\frac{6 k+2}{3}\right\rceil$, $e_{f}(0)=e_{f}(1)=e_{f}(2)-1=3 k$ if $k$ is even and $v_{f}(0)+1=v_{f}(1)=v_{f}(2)=$ $\left\lceil\frac{6 k+2}{3}\right\rceil, e_{f}(0)=e_{f}(1)-1=e_{f}(2)=3 k$ if $k$ is odd.

Case (ii): $n \equiv 1(\bmod 3), n=3 k+1, k>1$.

For $\mathrm{k}$ is even, $\mathrm{f}\left(\mathrm{u}_{i}\right)= \begin{cases}0 & \text { if } 1 \leq i \leq k+1 \\ 2 & \text { if } k+2 \leq i \leq\left\lceil\frac{3 k+1}{2}\right\rceil \\ 1 & \text { if }\left\lceil\frac{3 k+1}{2}\right\rceil+1 \leq i \leq 3 k+1\end{cases}$

and $f\left(v_{i}\right)= \begin{cases}0 & \text { if } 1 \leq i \leq k \\ 1 & \text { if } k+2 \leq i \leq\left\lceil\frac{3 k+1}{2}\right\rceil \\ 2 & \text { if } i=k+1 \text { and }\left\lceil\frac{3 k+1}{2}\right\rceil+1 \leq i \leq 3 k+1\end{cases}$ 
For $k$ is odd, $f\left(u_{i}\right)= \begin{cases}0 & \text { if } 1 \leq i \leq k+1 \\ 2 & \text { if } k+2 \leq i \leq\left\lceil\frac{3 k+1}{2}\right\rceil \\ 1 & \text { if }\left\lceil\frac{3 k+1}{2}\right\rceil+1 \leq i \leq 3 k+1\end{cases}$

and $\mathrm{f}\left(\mathrm{v}_{i}\right)= \begin{cases}0 & \text { if } 1 \leq i \leq k \\ 1 & \text { if } k+1 \leq i \leq\left\lceil\frac{3 k+1}{2}\right\rceil \\ 2 & \text { if }\left\lceil\frac{3 k+1}{2}\right\rceil+1 \leq i \leq 3 k+1\end{cases}$

For $k=1, f\left(u_{1}\right)=f\left(u_{2}\right)=f\left(v_{1}\right)=0, f\left(v_{2}\right)=f\left(u_{4}\right)=1, f\left(u_{3}\right)=$ $f\left(v_{3}\right)=f\left(v_{4}\right)=2$.

From the above labeling we have, $v_{f}(0)+1=v_{f}(1)=v_{f}(2)+1=$ $\left\lceil\frac{6 k+4}{3}\right\rceil, e_{f}(0)-1=e_{f}(1)=e_{f}(2)=3 k+1$ if $k$ is odd and $v_{f}(0)+1=$ $v_{f}(1)+1=v_{f}(2)=\left\lceil\frac{6 k+4}{3}\right\rceil, e_{f}(0)-1=e_{f}(1)=e_{f}(2)=3 k+1$ if $k$ is even and $k=1$.

Case (iii): $n \equiv 2(\bmod 3), n=3 k+2$ and $k$ is even.

$\mathrm{f}\left(\mathrm{u}_{i}\right)= \begin{cases}0 & \text { if } 1 \leq i \leq k+1 \\ 2 & \text { if } k+2 \leq i \leq \frac{3 k+2}{2} \\ 1 & \text { if } \frac{3 k+2}{2}+1 \leq i \leq 3 k+2\end{cases}$

and $f\left(v_{i}\right)= \begin{cases}0 & \text { if } 1 \leq i \leq k+1 \\ 1 & \text { if } k+2 \leq i \leq \frac{3 k+2}{2} \\ 2 & \text { if } \frac{3 k+2}{2}+1 \leq i \leq 3 k+2 .\end{cases}$

From the above labeling we have, $v_{f}(0)=v_{f}(1)=v_{f}(2)=2 k+2, e_{f}(0)-$ $1=e_{f}(1)=e_{f}(2)=3 k+2$. Hence, we have $\left|v_{f}(i)-v_{f}(j)\right| \leq 1$ and $\left|e_{f}(i)-e_{f}(j)\right| \leq 1$ for all $i, j=0,1,2$. Thus, $f$ is a 3 -product cordial labeling. Therefore, $K_{1, n} \times K_{2}$ is a 3 -product cordial graph.

An example for the 3 -product cordial labeling for the book graph $K_{1,7} \times$ $K_{2}$ is shown in Figure 3. 


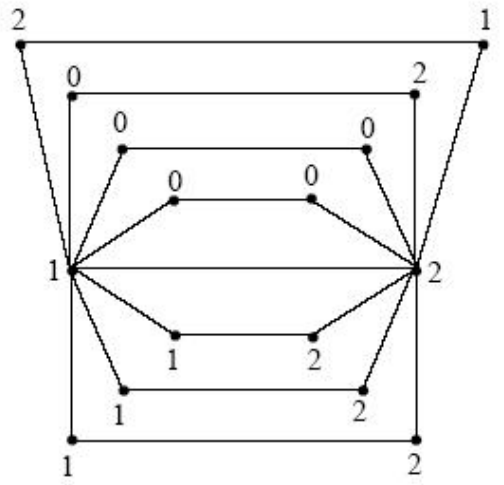

Figure 3.

Theorem 2.4. The graph $P\left(K_{2}+m K_{1}, I\right)$ is a 3-product cordial graph if and only if $m \equiv 2(\bmod 3)$.

Proof. Let $V\left(P\left(K_{2}+m K_{1}, I\right)\right)=\left\{u, u^{\prime}, v, v^{\prime}, u_{i}, v_{i} / 1 \leq i \leq m\right\}$ and $E\left(P\left(K_{2}+m K_{1}, I\right)\right)=\left\{u u_{i}, u^{\prime} u_{i}, v v_{i}, v^{\prime} v_{i}, u u^{\prime}, v v^{\prime}, u^{\prime} v, u v^{\prime}, u_{i} v_{i} / 1 \leq i \leq\right.$ $m\}$. Then $\left|V\left(P\left(K_{2}+m K_{1}, I\right)\right)\right|=2 m+4$ and $\left|E\left(P\left(K_{2}+m K_{1}, I\right)\right)\right|=5 m+4$.

Define a vertex labeling $f: V\left(P\left(K_{2}+m K_{1}, I\right)\right) \rightarrow\{0,1,2\}$ by $f(u)=$ $1, f\left(u^{\prime}\right)=2, f(v)=2, f\left(v^{\prime}\right)=1$.

$$
\begin{aligned}
\mathrm{f}\left(\mathrm{u}_{i}\right)= \begin{cases}0 & \text { for } 1 \leq i \leq\left\lceil\frac{m}{3}\right\rceil \\
1 & \text { for } i=\left\lceil\frac{m}{3}\right]+j \text { if } j \equiv 1(\bmod 2) \\
2 & \text { for } i=\left[\frac{m}{3}\right\rceil+j \text { if } j \equiv 0(\bmod 2)\end{cases} \\
\text { and } f\left(v_{i}\right)= \begin{cases}0 & \text { for } 1 \leq i \leq\left\lceil\frac{m}{3}\right] \\
1 & \text { for } i=\left\lceil\frac{m}{3}\right]+j \text { if } j \equiv 0,3(\bmod 4) \\
2 & \text { for } i=\left\lceil\frac{m}{3}\right]+j \text { if } j \equiv 1,2(\bmod 4) \text {. }\end{cases}
\end{aligned}
$$

In view of the above labeling pattern, we have $v_{f}(0)+1=v_{f}(1)=$ $v_{f}(2)=\left\lceil\frac{2 m+4}{3}\right\rceil, e_{f}(0)=e_{f}(1)+1=e_{f}(2)=\left\lceil\frac{5 m+4}{3}\right\rceil$ if $m$ is even and $v_{f}(0)+1=v_{f}(1)=v_{f}(2)=\left\lceil\frac{2 m+4}{3}\right\rceil, e_{f}(0)=e_{f}(1)=e_{f}(2)+1=$ $\left\lceil\frac{5 m+4}{3}\right\rceil$ if $m$ is odd. Thus, we have $\left|v_{f}(i)-v_{f}(j)\right| \leq 1$ and $\mid e_{f}(i)-$ $e_{f}(j) \mid \leq 1$ for all $i, j=0,1,2$. Hence, $f$ is a 3 -product cordial labeling of $P\left(K_{2}+m K_{1}, I\right)$ if $m \equiv 2(\bmod 3)$. 
Conversely, we assume that $m \equiv 0(\bmod 3)$ and take $m=3 k$. Then $\left|V\left(P\left(K_{2}+m K_{1}, I\right)\right)\right|=6 k+4$ and $\left|E\left(P\left(K_{2}+m K_{1}, I\right)\right)\right|=15 k+4$. Let $f$ be a vertex 3 -product cordial labeling of $P\left(K_{2}+m K_{1}, I\right)$. Then $v_{f}(0)-$ $v_{f}(1)-1=v_{f}(2)=2 k+1$ or $v_{f}(0)=v_{f}(1)=v_{f}(2)-1=2 k+1$ and $e_{f}(0)=5 k+1$ or $5 k+2$. If $f(u), f\left(u^{\prime}\right), f(v), f\left(v^{\prime}\right)$ are zero and the remaining $2 k-3$ vertices are either $f\left(u_{i}\right)=0$ for $1 \leq i \leq k-2 k>2$ and $f\left(v_{i}\right)=0$ for $1 \leq i \leq k-1, k>2$ or $f\left(u_{i}\right)=0$ for $1 \leq i \leq k-1, k>2$ and $f\left(v_{i}\right)=0$ for $1 \leq i \leq k-2, k>2$ then $e_{f}(0)=13 k+3$. If one of $f(u)$ or $f\left(u^{\prime}\right)$ and $f(v)$ or $f\left(v^{\prime}\right)$ is zero and the remaining $2 k-1$ vertices are either $f\left(u_{i}\right)=0$ for $1 \leq i \leq k-1, k>1$ and $f\left(v_{i}\right)=0$ for $1 \leq i \leq k, k>1$ or $f\left(u_{i}\right)=0$ for $1 \leq i \leq k, k>1$ and $f\left(v_{i}\right)=0$ for $1 \leq i \leq k-1, k>1$ then $e_{f}(0)=7 k+3$ or $7 k+4$. If all the $2 k+1$ vertices are either $f\left(u_{i}\right)=0$ for $1 \leq i \leq k+1$ and $f\left(v_{i}\right)=0$ for $1 \leq i \leq k$ or $f\left(u_{i}\right)=0$ for $1 \leq i \leq k$ and $f\left(v_{i}\right)=0$ for $1 \leq i \leq k+1$ then $e_{f}(0)=5 k+3$. Hence none of $f(u), f\left(u^{\prime}\right), f(v)$ and $f\left(v^{\prime}\right)$ is zero. From the above arguments, we get a contradiction to, $f$ is a 3 -product cordial labeling. Hence, $P\left(K_{2}+m K_{1}, I\right)$ is not a 3-product cordial graph if $m \equiv 0(\bmod 3)$.

We assume that $m \equiv 1(\bmod 3)$ and take $m=3 k+1$. Then $\mid V\left(P\left(K_{2}+\right.\right.$ $\left.\left.m K_{1}, I\right)\right) \mid=6 k+6$ and $\left|E\left(P\left(K_{2}+m K_{1}, I\right)\right)\right|=15 k+9$. Let $f$ be a vertex 3product cordial labeling of $P\left(K_{2}+m K_{1}, I\right)$. Hence we have $v_{f}(0)=v_{f}(1)=$ $v_{f}(2)=2 k+2$ and $e_{f}(0)=e_{f}(1)=e_{f}(2)=5 k+3$. If $f(u), f\left(u^{\prime}\right), f(v), f\left(v^{\prime}\right)$ are zero and the remaining $2 k-2$ vertices are $f\left(u_{i}\right)=f\left(v_{i}\right)=0$ for $1 \leq i \leq k-1, k>1$ then $e_{f}(0)=13 k+7$. If one of $f(u)$ or $f\left(u^{\prime}\right)$ and $f(v)$ or $f\left(v^{\prime}\right)$ is zero and the remaining $2 k$ vertices are $f\left(u_{i}\right)=f\left(v_{i}\right)=0$ for $1 \leq i \leq k$ then $e_{f}(0)=9 k+6$ or $9 k+5$. If all the $2 k+2$ vertices are $f\left(u_{i}\right)=f\left(v_{i}\right)=0$ for $1 \leq i \leq k+1$ then $e_{f}(0)=5 k+5$. Hence none of $f(u), f\left(u^{\prime}\right), f(v)$ and $f\left(v^{\prime}\right)$ is zero. From the above arguments, we get a contradiction to, $f$ is a 3 -product cordial labeling. Hence, $P\left(K_{2}+m K_{1}, I\right)$ is not a 3 -product cordial graph if $m \equiv 1(\bmod 3)$.

An example for the 3 -product cordial labeling for the graph $P\left(K_{2}+\right.$ $\left.5 K_{1}, I\right)$ is shown in Figure 4. 


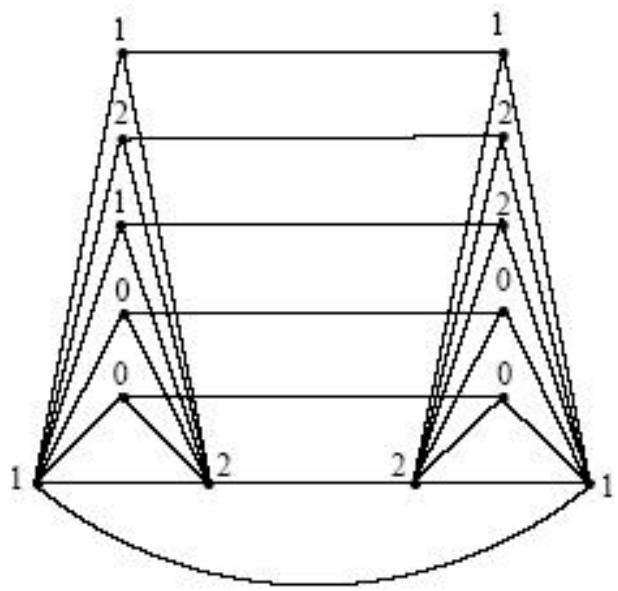

Figure 4.

\section{References}

[1] I. Cahit, Cordial Graphs: A weaker version of graceful and harmonious graphs, Ars Combinatoria, 23, pp. 201-207, (1987).

[2] Joseph A. Gallian, A dynamic survey of graph labeling, The Electronic Journal of Combinatorics, (2018) \# DS6.

[3] F. Harary, Graph Theory, Addision Wesley, Massachusetts, (1972).

[4] P. Jeyanthi and A. Maheswari, 3-product cordial labeling of some graphs, International Journal on Mathematical Combinatorics, 1, pp. 96-105, (2012).

[5] P. Jeyanthi and A. Maheswari, 3-product cordial labeling, SUT Journal of Mathematics, 48, pp. 231-240, (2012).

[6] P. Jeyanthi and A. Maheswari, 3-product cordial labeling of star graphs, Southeast Asian Bulletin of Mathematics, 39, pp. 429-437, (2015).

[7] P. Jeyanthi and A. Maheswari, Some results on 3-product cordial labeling, Utilitas Mathematica, 99, pp. 215-229, March, (2016). 
[8] P. Jeyanthi, A. Maheswari and M. Vijayalakshmi, 3-Product cordial labeling of some snake graphs, Proyecciones Journal of Mathematics, 38(1), Vol. 38, No. 1, pp. 13-30, March, (2019).

[9] R. Ponraj, M. Sivakumar and M. Sundaram, $k$-product cordial labeling of graphs, Int.J. Contemp. Math. Sciences, 7, (15), pp. 733-742, (2012).

[10] M. Sundaram, R. Ponraj and S. Somasundaram, Product Cordial labeling of graphs, Bulletin of Pure and Applied Sciences, 23E(1), pp. 155-163, (2004).

[11] M. Sundaram, R. Ponraj and S. Somasundaram, EP-cordial labeling of graphs, Varahmihir Journal of Mathematical Sciences, 7(1), pp. 183-194, (2007).

\section{P. Jeyanthi}

Research Centre, Department of Mathematics

Govindammal Aditanar College for Women

Tiruchendur - 628 215,

Tamilnadu,

India

e-mail: jeyajeyanthi@rediffmail.com

\section{A. Maheswari}

Department of Mathematics

Kamaraj College of Engineering and Technology

Virudhunagar - 626 001,

Tamilnadu,

India

e-mail: bala_nithin@yahoo.co.in

and

\section{Vijayalakshmi}

Department of Mathematics

Dr. G. U. Pope College of Engineering

Sawyerpuram, Thoothukudi District

Tamilnadu,

India

e-mail: viji_mac@rediffmail.com 